\title{
ARTICLES
}

\section{IMPLEMENTATION OF THE NATIONAL DIABETES EDUCATION PROGRAM TO IMPROVE DIABETES SELF-MANAGEMENT IN HISPANIC PATIENTS}

Ms. Neena Mathew*

*APRN. FNP-BC, PMHNP-BC

DOI: http://doi.org/10.47211/tg.2021.v08i04.004

\section{ABSTRACT}

Diabetes mellitus (DM) is a global, costly, and increasing illness. Type 2 diabetes (DM2) can be managed with lifestyle changes, such as better diet choices. In the United States, Hispanic individuals are at high risk for DM2 and have the third highest prevalence of DM2 among all ethnicities. Polk County, Florida, has a statistically higher prevalence of DM2 residents than other Florida counties. Almost one-quarter of the population are Hispanic, and many live below the poverty line, are uninsured, and are illegal immigrants. At Parkview Medical Clinic, a free clinic in Polk County, $80 \%$ of the patients have DM2. The goal of this project was to help these patients improve their dietary habits and blood glucose levels with the NDEP Program, 4 Steps to Manage Your Diabetes for Life. The theoretical framework was Orem's self-care theory, and the Plan-Do-Check-Act change model was used. After training by the project director, 10 volunteer nurse practitioners (NPs) individually instructed 37 patients weekly for 20 to 30 minutes over 9 weeks. In Weeks 1 and 9, the NPs administered the Diabetes Self-Management Questionnaire (DSMQ) and measured patients' blood glucose levels. The results were charted, and data were analyzed with paired samples $t$ tests. Results indicated that patients improved significantly in their diet choices and blood glucose levels ( $p$ <.001) from pretest (37 participants) to posttest (19 participants). Follow-ups at 3-and 6 months were recommended, and implications for nursing included greater use of the NDEP Program and ongoing patient education on self-management of diabetes.

Key words: blood glucose, diabetes, food choices, NDEP Program, nurse provider, self-care, self-management.

\section{ABOUT AUTHOR:}

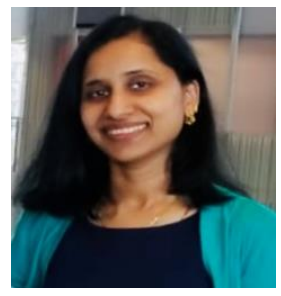

Ms. Mathew is a Board Certified Nurse Practitioner who is qualified to practice in both primary care and psychiatry; diagnose, treat, and prescribe medication for across the life span including children, adolescents, adults and older adults. She is currently working as a Lead Nurse Practitioner with Park Place Behavioral Healthcare, since 2016, which is a community mental health care facility in Orlando Florida. Some of her practice specialties include (but not limited to) ADHD, Depression, Anxiety and Bipolar.

Mathew's style of practice can be traditional (prescribing up to date psychiatric medications) or alternative medicine (natural treatments including but not limited to vitamins, supplements, lifestyle modification, nutrition, exercise and stress management). She believes in the mind/body/spiritual connection, and we are made up as a whole person and not a series of parts, just as Hippocrates "The Father of Medicine." 


\section{ARTICLES}

Diabetes mellitus (DM) is a common, chronic, and costly metabolic disease which currently affects millions of people in the United States and worldwide (Centers for Disease Control and Prevention [CDC], 2017). According to the World Health Organization (WHO, 2018), the number of people diagnosed with type 2 DM has increased four times since the 1980s. The WHO (2018) encouraged control and prevention of DM and its complications by promotion of healthy diets, consistent physical activity, avoidance of tobacco use, and maintenance of a healthy body weight. Polk County had a statistically higher prevalence of the population diagnosed with DM compared to other counties in Florida (Florida Diabetes Report, 2017). The Parkview Medical Clinic in Haines City, Polk County, is a free community clinic, especially for patients $200 \%$ below the poverty level (Parkview Medical Clinic, 2019a). The Hispanic patients who receive care and treatment at the clinic are from low-income households, undocumented immigrants, and uninsured. Approximately 80\% have type 2 diabetes (Parkview Medical Clinic, 2019b).

\section{Objective}

Two aims were identified for the project:

- To help adult Hispanic patients diagnosed with DM2 improve their dietary choices and blood glucose levels with implementation of the 4 Steps to Manage Your Diabetes for Life Program (NDEP, 2016).

- To implement and sustain practice change at Parkview Medical Clinic by increasing the NPs' awareness of DM2 patients' self-management techniques and improving the patients' awareness and implementation of self-management of their diet choices and blood glucose levels.

The objectives of the project were as follows:

- To train NPs at Parkview Medical Clinic to administer a diabetes education program.

- To educate patients at Parkview Medical Clinic with evidence-based procedures to reduce their challenges to effective diabetes care.

- To promote better quality of life among adult DM2 Hispanic patients at Parkview Medical Clinic through healthier dietary choices and blood glucose management.

- To provide evidence-based data about the effectiveness of the use of the NDEP 4 Steps to Manage Your Diabetes for Life Program.

\section{Methods}

Orem's (1971) self-care theory and the Plan-Do-Check-Act (PDCA) change model (Johnson, 2016; Leis \& Shojania, 2017) were used to guide the project. Ten nurse practitioners volunteered at Parkview Medical Clinic. Forty patients with DM2 were chosen by the NPs and the DNP student after chart examination, with 37 participating in the pretest and 19 in the posttest. The project manager first educated the NPs on the project purpose, implementation, traditional Hispanic foods and customs, and the 4 Steps Program. Materials were distributed. Each provider was assigned three to four patients and followed up with the assigned patients, working with them throughout the 9 weeks. In the weekly meetings of 20 to 30 minutes, with the 4 Steps as a guide, the NPs asked patients about their grocery choices, cooking, meal choices, and diets, considering the Hispanic traditional foods. The NPs also instructed the patients in completing a food choice survey, the Diabetes Self-Management Questionnaire (DSMQ), and blood glucose charts at weeks 1 and 9. The NPs kept records of the patients' DSMQ results and blood glucose levels.

Data analysis took place first with descriptive calculation of frequencies and percentages of patients' responses pre- and post-implementation. Second, paired samples $t$ tests were used for determination of significant differences from Week 1 pre-intervention to Week 9 post intervention in the patients' food-choice survey items (DSMQ) and blood glucose readings.

\section{Results}

\section{Data Analysis Results}

The purpose of this project was for nurse practitioners (NPs) at the Parkview Medical Clinic to help adult Hispanic patients diagnosed with type 2 diabetes by using the 4 Steps to Manage Your Diabetes for Life Program (NDEP, 2016) to improve their dietary choices and blood glucose readings. Parkview Medical Clinic is in Polk County, Florida, which had a statistically higher prevalence of the population diagnosed with DM compared to other counties in Florida (Florida Diabetes Report, 2017). The Hispanic patients who receive care and treatment at the clinic are from low-income households. They are also undocumented immigrants, and uninsured. Approximately $80 \%$ of the patients have type 2 diabetes (Parkview Medical Clinic, 2019b). 


\section{ARTICLES}

\section{Methods and Procedures}

To address the PICOT question, the intervention was comprised of the NPs administered the survey to patients individually by pen and paper at the first and ninth weeks of the intervention. Administration took approximately 10 minutes per patient. The NPs measured the patients' blood glucose levels individually at the first and ninth weeks of the intervention, showing them also how to self-administer. This step took approximately 10 minutes per patient.

Use of the blood glucose chart also helped answer the PICOT question directly. Patients' blood glucose readings were measured and recorded in Week 1 and Week 9. The results were compared statistically, and each patient's progress in glucose regulation was evaluated. Descriptive and inferential statistics were used. The descriptive statistics (frequencies and percentages) described the sample before and after the intervention. Frequencies and percentages are a basic statistical method for describing a sample (Gray, Grove, \& Sutherland, 2017). The inferential statistical methods chosen were two paired samples $t$ tests. Statistical significance was tested at the generally accepted level for research, $p<.05$. A paired samples $t$ test is appropriate when assessing for differences on a continuous variable between two points in time (Pallant, 2013).

\section{Major Findings}

To address the PICOT question, two paired samples $t$ tests were conducted to examine for differences in clinics' type 2 diabetics' food choices and blood glucose readings before and after the intervention. For the food choice measurement, the sample size consisted of 19 participants who completed both the pretest and posttest. For the blood glucose reading, the sample size consisted of 19 participants who completed both the pretest and posttest. Prior to analysis, the assumptions of a paired samples $t$ test were assessed: absence of outliers and a normal distribution for the differences in the dependent variables. Potential outliers were checked through use of standardized values, or $z$-scores. Z-scores exceeding the threshold \pm 3.29 standard deviations are typically considered outliers and are subsequently removed (Tabachnick \& Fidell, 2013). However, none of the values for food choices or blood glucose readings exceeded the outlier threshold. Therefore, no scores were removed in the analysis. Normality was tested with a Kolmogorov-Smirnov test on the difference measurements. The assumption of normality was met for food choices $(p=.200)$ and blood glucose readings $(p=.200)$.

A paired samples $t$ test was conducted for food choices with the DSME pretest and posttest. The result of the paired samples $t$ test was significant for food choices scores, $t(10)=-7.03, p<.001$, suggesting that there was a significant increase in healthy food choices scores between pretest and posttest. This result indicates that implementation of the evidence-based program improved participants' food choices.

A paired samples $t$ test was conducted for blood glucose readings with the blood glucose chart. The result of the paired samples $t$ test was significant for blood glucose readings, $t(18)=13.74, p<.001$, suggesting that the there was a significant decrease in blood glucose scores between pretest and posttest. This result indicates that implementation of the evidence-based program improved participants' blood glucose readings.

The purpose of this project was for nurse practitioners (NPs) at the Parkview Medical Clinic to help adult Hispanic patients diagnosed with type 2 diabetes by using the 4 Steps to Manage Your Diabetes for Life Program (NDEP, 2016) to improve their dietary choices and blood glucose readings. In this chapter, the findings of the data analysis were presented. Findings of the inferential analyses indicated that there were significant improvements in food choices and blood glucose readings after the intervention. In the next chapter, the findings of the data analysis will continue to be explored in connection with the literature.

\section{Conclusions}

The NDEP Program (NDEP, 2016) was effective in helping DM2 patients manage their diabetes by improving their diet choices and lowering their blood glucose. Follow-ups should take place at 3-and 6-month intervals, with additional educational activities facilitated by the NPs, such as lectures, exercises, group sharing, and inclusion of family members. Implications for nursing practice include NP instruction in Spanish and the Hispanic culture and training in the NDEP Program, support groups for patients and their families, and community outreach on diabetes self-management with lectures, brochures, radio announcements, and other media.

\section{Ethics and Human Subjects Protection}

Patients' human rights were protected, and the privacy of their health information ensured with data collection by the NPs' assignment to patients by number only. No patient names were used, and results were reported in group 


\section{ARTICLES}

form only. The patient informed consent form (see Appendix $\mathrm{H}$ ) assured patients of these rights, and the NPs also ensured that patients understood the form at their first visit and answered any questions.

The data in paper format were kept in a secured file cabinet in the student's home office. Only the student had access to these data. The electronic data were stored on the student's secure computer at her workplace with an encryption accessible only to the student and IT personnel. On project completion, the results were shared with the clinic, and the data were transferred to the student's secure password-protected home computer, to which she alone has access. The IT personnel deleted the information from the student's workplace computer. The data will be saved for 7 years on the student's password-protected home computer, per university requirements, and then appropriately destroyed.

\section{Implications for Nursing}

The results of this project suggested that an evidence-based educational initiative can enable nurse practitioners to help DM2 patients significantly increase their healthier food choices and decrease the blood glucose levels. Further, for such improvements, outpatient clinical settings must make necessary changes to educate patients to improve their diet choices and portion sizes and lower blood glucose readings.

\section{REFERENCES}

1. American Diabetes Association. (2018). Statistics about diabetes. Retrieved from http://www. diabetes.org/diabetes-basics/statistics/

2. Buckley, J., Yekta, S., Joseph, V., Johnson, H., Oliverio, S., \& Groot, A. (2015). Vida Sana: A lifestyle intervention for uninsured, predominantly Spanish-speaking immigrants improves metabolic syndrome indicators. Journal of Community Health, 40(1), 116-123. https://doi.org/10.1007/s10900-014-9905-z

3. Centers for Disease Control and Prevention. (2017). National diabetes statistics report, 2017.

4. Devchand, R., Nicols, C., Gallivan, J. M., Tiktin, M., Krause-Steinrauf, H., Larkin, M., ...

5. GRADE Research Group. (2017). Assessment of a National Diabetes Education Program diabetes management booklet: The GRADE experience. Journal of the American Association of Nurse Practitioners, 29(5), 255-263. Retrieved from https://onlinelibrary.wiley .com/doi/abs/ 10.1002/ 2327-6924.12445

6. Florida Diabetes Advisory Council. (2017). Florida diabetes report. Retrieved from

7. http://www.floridahealth.gov/provider-and-partner-resources/dac/_documents/dac-reportjanuary 2017.pdf

8. Gray, J. R., Grove, S. K., \& Sutherland, S. (2017). Burns and Groves' the practice of nursing research: Appraisal, synthesis, and generation of evidence (8th ed.). St. Louis, MO: Elsevier.

9. International Diabetes Federation. (2017). Diabetes facts and figures. Retrieved from

10. https://www.idf.org/aboutdiabetes/what-is-diabetes/facts-figures.html

11. Johnson, C. N. (2016). The benefits of PDCA. Quality Progress, 49(1), 45. Retrieved from

12. https:// chamberlainuniversity .idm.oclc.org /login?url=https://search-proquest- com. chamberlainuniversity .idm.oclc.org /docview /1762043658? accountid=147674

13. Kouzes, J. M., \& Posner, B. Z. (2016). Learning leadership: The five fundamentals of becoming

14. an exemplary leader. New York, NY: John Wiley.

15. Langabeer, J. R. (2018). Performance management methods and tools. In J. R. Langabeer (Ed.).,

16. Performance improvement in hospitals and health systems (2nd ed., pp. 43-62). Boca Raton, FL: CRC Press.

17. Leis, J. A., \& Shojania, K. G. (2017). A primer on PDSA: Executing plan-do-study-act cycles

18. in practice, not just in name. BMJ Quality and Safety, 26(7), 572-577.

19. Mehravar, F., Mansournia, M. A., Holakouie-Naieni, K., Nasli-Esfahani, E., Mansournia, N., \&

20. Almasi-Hashiani, A. (2016). Associations between diabetes self-management and microenterprise owners' vascular complications in patients with type 2 diabetes. Epidemiology and Health, 38, 1-6.

21. National Diabetes Education Program. (2016). 4 steps to manage your diabetes for life.

22. Washington, DC: U.S. Department of Health and Human Services, National Institute of 


\section{ARTICLES}

Diabetes and Digestive and Kidney Diseases. Retrieved from https://www.niddk.nih.gov/healthinformation/diabetes/overview/managing-diabetes/4-steps

23. Orem, D. (1971). Nursing: Concepts of practice. New York, NY: McGraw-Hill.

24. Pallant, J. (2013). SPSS survival manual (5th ed.). New York, NY: McGraw-Hill

25. Parkview Medical Clinic. (2019a). Health information. Retrieved fromhttp://parkviewmedicalclinic.org/develop/category/health-information/

26. Parkview Medical Clinic. (2019b). Patients. Retrieved from http://parkviewmedicalclinic.org/develop/patients/Parkview Medical Clinic

27. Parkview Medical Clinic. (2019c). Volunteers. Retrieved from http://parkviewmedicalclinic.org/develop/volunteers/

28. Parkview Medical Clinic. (2019d). Welcome to Parkview Medical Clinic. Retrieved from http://www.parkviewmedicalclinic.org/

29. Ramal, E., Champlin, A., \& Bahjri, K. (2018). Impact of a plant-based diet and support on mitigating type 2 diabetes mellitus in Latinos living in medically underserved areas. American Journal of Health Promotion, 32(3), 753-762. https://doi.org/10.1177/0890117117706793

30. Schmitt, A., Gahr, A., Hermanns, N., Kulzer, B., Huber, J., \& Haak, T. (2013). The Diabetes_SelfManagement Questionnaire (DSMQ): Development and evaluation of an instrument to assess diabetes self-care activities associated with glycemic control. Health and Quality of Life Outcomes, 11(1), 1-14. doi:10.1186/1477-7525-11-138. Retrieved from https://www.ncbi.nlm.nih.gov/pmc/articles/PMC3751743/

31. Schmitt, A., Reimer, A., Hermanns, N., Huber, J., Ehrmann, D., Schall, S., \& Kulzer, B. (2016).

32. Assessing diabetes self-management with the diabetes self-management questionnaire (DSMQ) can help analyse behavioural problems related to reduced glycaemic control. PloS one, 11(3), e0150774.

33. Siminerio, L. M., Albright, A., Fradkin, J., Gallivan, J., McDivitt, J., Rodríguez, B., . . .

34. Wong, F. (2018). The National Diabetes Education Program at 20 years: Lessons learned and plans for the future. Diabetes Care, 41(2), 209-218.

35. Tabachnick, B. G., \& Fidell, L. S. (2013). Using multivariate statistics (6th ed.). Boston, MA:

36. Allyn \& Bacon. Thojampa, S., \& Mawn, B. (2017). The moderating effect of social cognitive factors on self-management activities and $\mathrm{HbA1c}$ in Thai adults with type-2 diabetes. International Journal of Nursing Sciences, 4(1), 34-37.

37. U.S. Department of Health and Human Services. (2016). Diabetes and Hispanic Americans. Retrieved from https://minorityhealth.hhs.gov/omh/browse.aspx?lvl=4\&lvlid=63

38. World Health Organization. (2018). 10 facts on diabetes. Retrieved from https://www.who.int/features/factfiles/diabetes/e 